\title{
Fabrication of High-Performance ZnO Thin-Film Transistors With Submicrometer Channel Length
}

\author{
Horng-Chih Lin, Senior Member, IEEE, Rong-Jhe Lyu, and Tiao-Yuan Huang, Fellow, IEEE
}

\begin{abstract}
A method was developed to fabricate $\mathrm{ZnO}$ thinfilm transistors (TFTs) with submicrometer channel length. In this scheme, mature process techniques are used to form a suspending hardmask bridge on the wafer surface, which enables the subsequent construction of a TFT by the sequential deposition of gate oxide, $\mathrm{ZnO}$ channel layer, and $\mathrm{Al}$ source/drain contacts. Excellent electrical characteristics were demonstrated by the fabricated $\mathrm{ZnO}$ TFTs that show high ON/OFF current ratio $\left(>10^{9}\right)$, low subthreshold swing $(89 \mathrm{mV} /$ decade), and high field-effect mobility $\left(41 \mathrm{~cm}^{2} / \mathrm{V} \mathrm{s}\right)$. Very small variation in the device characteristics is also demonstrated.
\end{abstract}

Index Terms-Metal oxide, shadow mask, submicrometer, thin-film transistors, $\mathrm{ZnO}$.

\section{INTRODUCTION}

$\mathbf{R}$ ECENTLY, metal oxide has been adopted as the channel material of thin-film transistors (TFTs) in various applications, such as active matrix liquid crystal displays [1], [2], high-frequency microwave devices [3], back-end-of-line power transistors [4], [5], and flexible displays [6]. In particular, $\mathrm{ZnO}$ and its variants, IGZO, HIZO, and so on have great potential to replace the conventional a-Si:H and ploy-Si materials due to their high mobility, high transparency, and low process temperature.

Several device structures of TFTs have been proposed and developed [7]. In 2010, Lu et al. [8] developed a simple and ingenious approach to fabricate coplanar ITO TFT using only one metal shadow mask. In this approach, the shadow mask was employed to define the source/drain (S/D) regions. After the sputtering deposition of an ITO film, thick S/D regions accompanied with a thin self-assembled channel are formed. The mechanism responsible for the above result is attributed to the scattering of the deposition species in the ambient.

Although the above scheme is simple and novel, there still exist a few issues.

Manuscript received July 3, 2013; accepted July 18, 2013. Date of publication August 15, 2013; date of current version August 21, 2013. This work was supported in part by the Ministry of Education in Taiwan under ATU Program, the NCTU-UCB I-RiCE program under Grant NSC-102-2911I-009-301, and the National Science Council under Contract NSC-99-2221-E009-167-MY3. The review of this letter was arranged by Editor W. S. Wong.

H.-C. Lin is with the Department of Electronics Engineering and Institute of Electronics, National Chiao Tung University, Hsinchu 300, Taiwan, and also with the National Nano Device Laboratories, Hsinchu 300, Taiwan (e-mail: hclin@faculty.nctu.edu.tw).

R.-J. Lyu and T.-Y. Huang are with the Department of Electronics Engineering and Institute of Electronics, National Chiao Tung University, Hsinchu 300, Taiwan.

Color versions of one or more of the figures in this letter are available online at http://ieeexplore.ieee.org.

Digital Object Identifier 10.1109/LED.2013.2274263 (a)

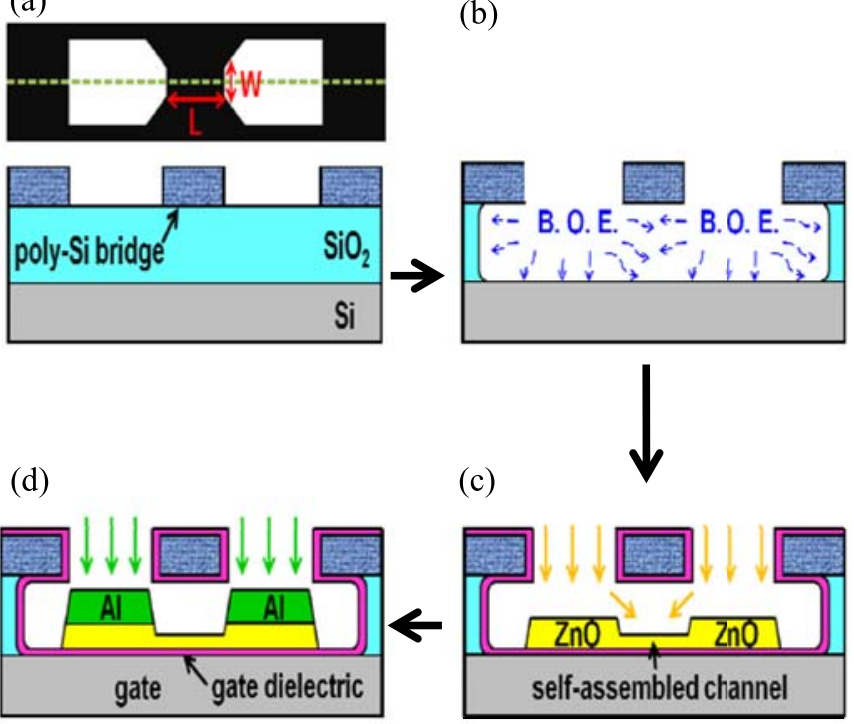

Fig. 1. Fabrication sequence of the $\mathrm{ZnO}$ TFT. Top view of the structure is shown at the top of (a). For simplicity, the deposited $\mathrm{ZnO}$ and $\mathrm{Al}$ on the poly-Si hardmask are not shown in (c) and (d).

1) Distance between the shadow mask and substrate is hard to control due to warps in both the shadow mask and substrate. This would result in a variation in the deposited profile and thus the device characteristics.

2) Dimensions of the patterns on the shadow mask are usually large (e.g., $\geqq 50 \mu \mathrm{m}$ ). Devices with submicrometer dimensions and beyond are difficult to achieve.

3) In the previous research [8] only one ITO film is deposited. This makes it hard to optimize the structure for a good TFT that contains insulating gate oxide, semiconducting channel, and metallic S/D contacts.

In this letter, a novel approach is proposed to address the above issues. The new scheme adopts mature lithographic and etching techniques so that submicrometer devices with small variations in characteristics can be readily achieved. Besides, we also show that the profile of the deposited films can be cleverly tailored. This feature favors the construction of refined device structures with improved characteristics.

\section{EXPERIMENTAL DETAILS}

The proposed scheme is demonstrated with a one-mask process. The fabrication sequence of the ZnO TFT is shown in Fig. 1. For simplicity, Si substrate is used as the bottom 

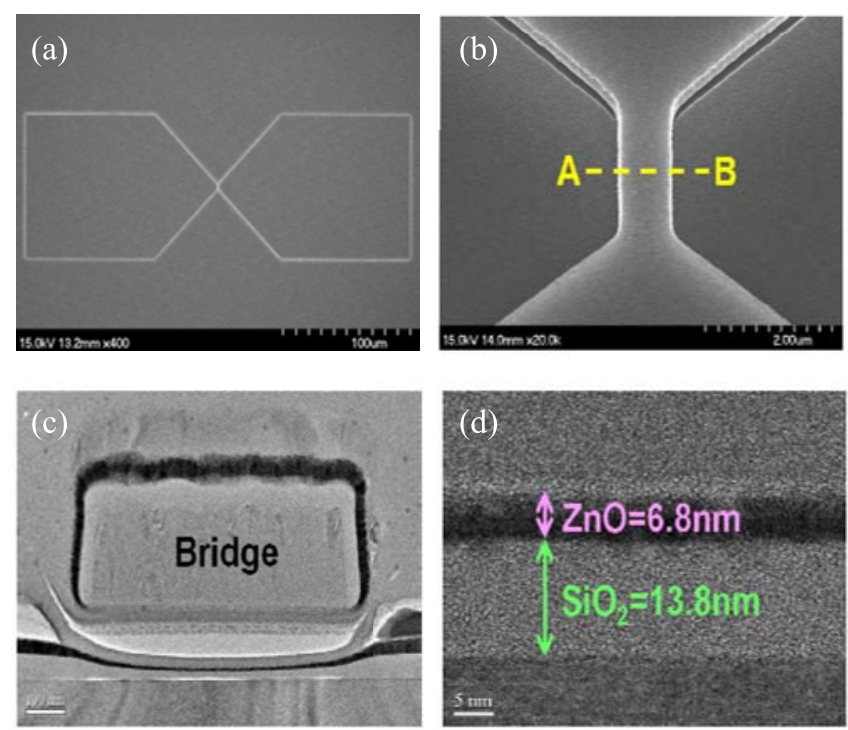

Fig. 2. (a) SEM top view image of a fabricated ZnO TFT. (b) Suspending bridge formed on the wafer surface. (c) TEM image showing the cross-section along the line $\mathrm{AB}$ in (b). In this figure a nitride capping layer was deposited to passivate and strengthen the device. (d) TEM image showing the thinnest part at the center of channel.

gate. First, a 400-nm-thick $\mathrm{SiO}_{2}$ and 200-nm-thick undoped ploy-Si were deposited sequentially on an n-type Si wafer by low-pressure chemical vapor deposition to serve as the sacrificial and hardmask layers, respectively. After an I-line-based photolithographic step to generate the photoresist pattern of S/D regions [top of Fig. 1(a)], the surface poly-Si was etched as shown in Fig. 1(a). Wet etching using buffer oxide etch solution was subsequently carried out to remove the underlying $\mathrm{SiO}_{2}$ [Fig. 1(b)]. After this step the central poly-Si stripe became a suspended bridge. A 50-nm-thick tetraethylorthosilicate (TEOS)-based $\mathrm{SiO}_{2}$ was then deposited by plasmaenhanced chemical vapor deposition (PECVD) at $300{ }^{\circ} \mathrm{C}$ under a pressure of 500 mtorr to serve as the gate dielectric, followed by the deposition of 50-nm-thick $\mathrm{ZnO}$ channel layer using a radio-frequency magnetron sputter at room temperature under a pressure of 5 mtorr [Fig. 1(c)]. The deposited $\mathrm{ZnO}$ film is polycrystalline as verified by the X-ray diffraction analysis. If the channel length (defined by the width of the poly-Si strip) is sufficiently short (e.g., $<0.8 \mu \mathrm{m}$ in the present case), a self-assembled channel is formed [8] that is much thinner than that at the S/D regions. Finally, 100-nm Al was deposited by a thermal coater at a pressure of $\sim 8 \times 10^{-6}$ torr to form the S/D contacts. Owing to the ultralow deposition pressure, the $\mathrm{Al}$ contacts are disconnected between the source and drain. Electrical characteristics of the ZnO TFTs were measured by an HP4156 parameter analyzer and the device structure was traced by scanning electron microscopy (SEM) and transmission electron microscopy (TEM).

\section{RESULTS AND DISCUSSION}

Fig. 2(a) and (b) shows the SEM top view images of a fabricated $\mathrm{ZnO}$ TFT. The suspending bridge is observed and its width is $\sim 0.8 \mu \mathrm{m}$ that is longer than the designed dimension $(0.6 \mu \mathrm{m})$. This is due to the follow-up deposition of gate
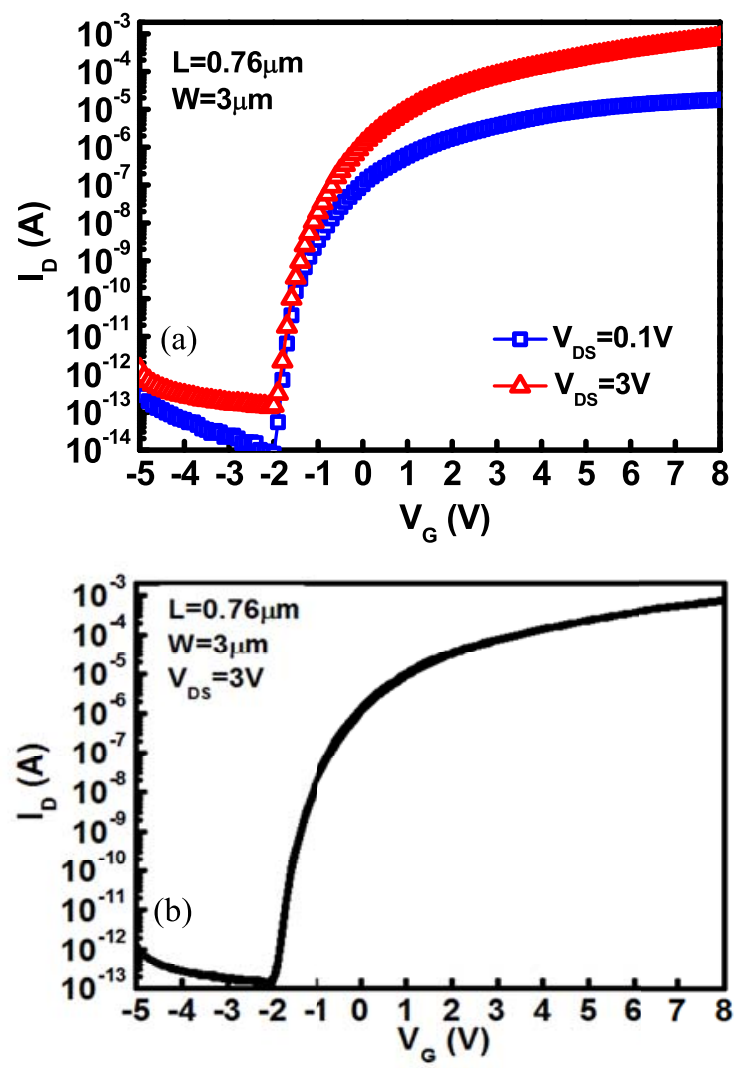

Fig. 3. (a) Transfer curves of a ZnO TFT at $V_{\mathrm{DS}}=0.1$ and 3 V. (b) Transfer curves of five devices located at different dies at $V_{\mathrm{DS}}=3 \mathrm{~V}$.

oxide, channel, and metal materials after the formation of suspending bridge. TEM image of the cross-section along the line $\mathrm{AB}$ in Fig. 2(b) is shown in Fig. 2(c). Continuous $\mathrm{ZnO}$ channel under the suspending bridge can be observed and its thickness is gradually thinned down along the direction toward the center of the channel. Similar condition can also be seen on the gate dielectric although to a lesser extent. In contrast, Al metal pads are isolated from each other and form the S/D regions. The cross-sectional image of a fabricated device is shown in Fig. 2(c). Obviously the profiles of the deposited films (gate oxide, $\mathrm{ZnO}$ channel, and $\mathrm{Al}$ S/D contact pads) are quite different and the differences are mainly related to the deposition pressure. As the scattering probability is greatly increased with increasing pressure, the film thickness is expected to be thicker at the channel center as the deposition pressure is higher. Fig. 2(d) shows the thinnest part of $\mathrm{ZnO}$ and oxide films at the center of the channel with the thickness of 6.5 and $13.8 \mathrm{~nm}$, respectively, which are much thinner than the set thickness $(50 / 50 \mathrm{~nm})$. As the deposition pressure of the PECVD process is two orders in magnitude higher than that of the sputtering process, the oxide is much thicker than the $\mathrm{ZnO}$ at the channel center. The deposition of $\mathrm{Al}$ film is prohibited in the central channel region underneath the suspended bridge owing to the fact that the process pressure is $<10^{-5}$ torr. Under such condition the scattering of the evaporated $\mathrm{Al}$ species in the chamber space is negligible.

Transfer curves of a typical $\mathrm{ZnO}$ TFT at $V_{\mathrm{DS}}=0.1$ and $3 \mathrm{~V}$ are shown in Fig. 3(a). The channel length of $\mathrm{ZnO}$ TFT is defined to be the distance between the two Al pads 


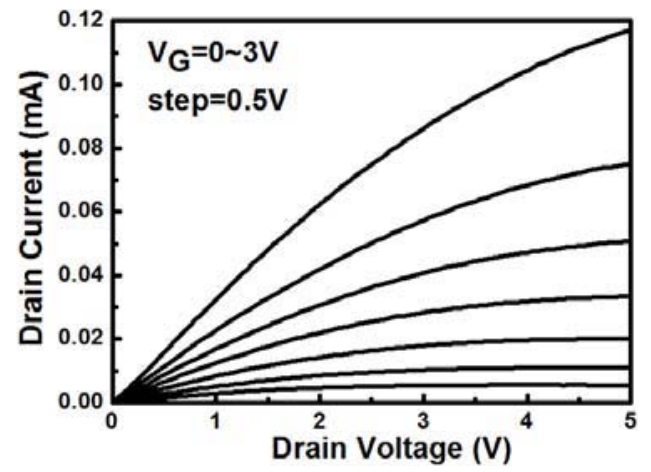

Fig. 4. Output characteristics of a $\mathrm{ZnO}$ TFT.

and is measured to be $0.76 \mu \mathrm{m}$ that is comparable with the planar dimension of the suspending poly-Si bridge. Excellent electrical characteristics are obtained in terms of high ON/OFF current ratio $>10^{9}$, good mobility value of $41 \mathrm{~cm}^{2} / \mathrm{V} \mathrm{s}$, and steep subthreshlod property with swing of $89 \mathrm{mV} /$ decade. The threshold voltage is $0.87 \mathrm{~V}$.

The variation of the device characteristics is also checked by measuring a number of devices on a wafer. Fig. 3(b) shows the transfer curves of five ZnO TFTs located on different dies of the wafer. Negligible differences are found among those characteristics, demonstrating good uniformity is achievable with the present fabrication approach. Fig. 4 shows the wellbehaved output characteristics of the device.

\section{CONCLUSION}

A new method employing mature semiconductor process techniques are developed in this letter to fabricate ZnO TFTs featuring submicrometer channel length, self-assembled channel layer, and self-aligned Al S/D contacts. Excellent transfer characteristics were achieved for the fabricated $\mathrm{ZnO}$ TFTs with very high ON/OFF current ratio $\left(>10^{9}\right)$, low subthreshold swing ( $89 \mathrm{mV} /$ decade), and high field-effect mobility $\left(41 \mathrm{~cm}^{2} / \mathrm{V} \mathrm{s}\right)$. Electrical properties of the manufactured devices are randomly selected from different dies on the wafer evidence, very small variation.

\section{REFERENCES}

[1] T. Hirao, T. Hiramatsu, T. Matsuda, et al., "Bottom-gate zinc oxide thin-film transistors (ZnO TFTs) for AM-LCDs," IEEE Trans. Electron Devices, vol. 55, no. 11, pp. 3136-3142, Nov. 2008.

[2] J. K. Jeong, "The status and perspectives of metal oxide thin-film transistors for active matrix flexible displays," Semicond. Sci. Technol., vol. 26, no. 3, p. 034008, Feb. 2011.

[3] B. Bayraktaroglu, K. Leedy, and R. Neidhard, "High-frequency ZnO thin-film transistors on Si substrates," IEEE Electron Device Lett., vol. 30, no. 9, pp. 946-948, Sep. 2009.

[4] S. Jeon, H. Kim, H. Choi, et al., "High performance bilayer oxide transistor for gate driver circuitry implemented on power electronic devices," in Proc. Symp. VLSI Technol., 2012, pp. $125-126$.

[5] K. Kaneko, N. Inoue, S. Saito, et al., "A novel BEOL transistor (BETr) with $\mathrm{InGaZnO}$ embedded in $\mathrm{Cu}$-interconnects for on-chip high voltage I/Os in standard CMOS LSIs," in Proc. Symp. VLSI Technol., 2011, pp. $120-123$.

[6] D. Zhao, D. A. Mourey, and T. N. Jackson, "Fast flexible plastic substrate ZnO circuits," IEEE Electron Device Lett., vol. 31, no. 4, pp. 323-325, Apr. 2010.

[7] M. Ito, C. Miyazaki, M. Ishizaki, et al., "Application of amorphous oxide TFT to electrophoretic display," J. Non-Cryst. Solids, vol. 354, pp. 2777-2782, May 2008.

[8] A. Lu, J. Sun, J. Jiang, et al., "One-shadow-mask self-assembled ultralow-voltage coplanar homojunction thin-film transistors," IEEE Electron Device Lett., vol. 31, no. 10, pp. 1137-1139, Oct. 2010 